\title{
Proliferation and adipogenic differentiation of human adipose-derived stem cells isolated from middle-aged patients with prominent orbital fat in the lower eyelids
}

\author{
Guang-Peng Liu, Cai-He Liao, Yi-Pin Xu \\ Department of Plastic and Reconstructive Surgery, Shanghai Tenth People's Hospital, Tongji University School of Medicine, Shanghai 200072, China.
}

Correspondence to: Dr. Guang-Peng Liu, Department of Plastic and Reconstructive Surgery, Shanghai Tenth People's Hospital, Tongji University School of Medicine, Shanghai 200072, China. E-mail: guangpengliu@163.com

How to cite this article: Liu GP, Liao $\mathrm{CH}, \mathrm{Xu}$ YP. Proliferation and adipogenic differentiation of human adipose-derived stem cells isolated from middle-aged patients with prominent orbital fat in the lower eyelids. Plast Aesthet Res 2016;3:322-7.

\section{Article history: \\ Received: 07-09-2016 \\ Accepted: $18-10-2016$ \\ Published: $25-10-2016$ \\ Key words: \\ Orbital fat, \\ adipose-derived stem cells, \\ progenitor cell number, \\ proliferation rate, \\ adipogenic differentiation}

\begin{abstract}
\end{abstract}
\section{INTRODUCTION}

Adipose-derived stem cells (ASCs) are a population of postnatal progenitor cells which exist abundantly in adipose tissue. These cells can expand rapidly in vitro, possess multi-lineage differentiation potential and remain stable over long-term culture. ${ }^{[1]}$ Compared to the adult stem cells derived from other tissue sources, such as bone marrow, muscle and synovium, advantages of utilizing ASCs include the large

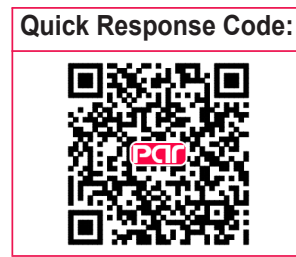


quantities of fat tissue, minimal patient discomfort, little donor-site morbidity and ease of cell isolation. ${ }^{[2]}$ Thus, there has been growing interest in applying ASCs as a potential cell source in the field of tissue engineering and regenerative medicine during the past decade.

The human body can be segmented into various depots of fatty tissue based on anatomic region. ${ }^{[3]}$ These depots vary in the amount of adipose tissue and may produce ASCs with different biological characteristics. The orbital cavity contains a highly specialized adipose depot which differs from the subcutaneous fat (SF) developmentally and functionally. ${ }^{[4]}$ The existence of human orbital adipose-derived stem cells (OASCs) also has been confirmed, which can differentiate into the corneal epithelial lineage, smooth muscle lineage and neuronal lineage under specific inductive conditions, showing great therapeutic potential in treating orbital and ocular diseases. ${ }^{[4-7]}$

The location of orbital fat (OF) may change dramatically from young to old age, especially in the lower eyelids. Normally the OF is located behind the septum and in front of the aponeurosis, occupying most of the orbital cavity. By middle or early old age, it often migrates forward to the preseptal region, resulting in a baggy appearance of the eyes. An interesting question therefore arises from this phenomenon: will aging influence the functional characteristics of OASCs? Identifying this issue is of significance when autologous OASC-based therapies are designed for elderly patients. To date, however, little literature has investigated the relationship between age and the biological properties of OASCs.

In this study, the authors tested whether the proliferative and differentiation potentials of human OASCs are affected by donor age. First, the OF samples were harvested from young (with normal OF) and middleaged patients (with protruded OF) during routine blepharoplasty. Histological evaluation was performed to observe the morphological changes of fat cells in relation to age. Next, OASCs were isolated from OF samples and expanded in vitro. The cell yield, surface marker expression and growth kinetics were assessed. Finally OASCs were cultured under adipogenic condition to compare their differentiation potential into adipocytes.

\section{METHODS}

\section{Harvest of orbital fat samples}

Adipose tissue samples were surgically harvested during lower lid blepharoplasty from the central compartments of the OF in 20 healthy female patients who had previously given informed consent. Patients with obesity (body mass index $>25 \mathrm{~kg} / \mathrm{m}^{2}$ ), orbital disease or endocrine disease were excluded from this study. The donors were divided into two age groups: group A (26.33 \pm 6.48 years old, $n=10)$ and group B (56.44 \pm 5.83 years old, $n=10)$. Patients in group $A$ had no baggy eye appearance while those in group $B$ had dermatochalasis with typical OF protrusion in the lower eyelids. Fat samples were preserved on ice under aseptic condition, transported to the laboratory immediately after surgery, and processed within $6 \mathrm{~h}$.

\section{Evaluation of adipocyte morphology}

After weight measurement, four specimens in each group were fixed in $10 \%$ formalin overnight, embedded in paraffin, sectioned in 5- $\mu \mathrm{m}$ thickness and processed for routine hematoxylin and eosin (HE) staining. Images were taken in triplicate for each specimen using an optical microscope (IX70, Olympus, Tokyo, Japan). The photographs were processed with the green channel to enhance edge prominence using the Image-Pro Plus software (v6.0, Media Cybernetics, Silver Spring, MD, USA). Three main descriptive parameters were measured for evaluation of adipocyte morphology as follows: cell diameter (length of the longest line joining two points and passing through the centroid), perimeter and area. ${ }^{[8]}$ Approximately 300 adipocytes were measured for each histological image.

\section{Isolation and expansion of OASCs}

OASCs were isolated and expanded using methods previously reported with minor modification. ${ }^{[4]}$ Briefly, after washing in phosphate buffer solution (PBS, pH 7.4, Sigma, Shanghai, China) extensively, 6 OF samples from each group were minced with sterile scissors and digested with $0.1 \%$ type I collagenase solution (Worthington Biochemical Corp, Lakewood, $\mathrm{NJ}, \mathrm{USA}$ ) at $37^{\circ} \mathrm{C}$ for 60 min with constant agitation. The upper layer of adipocytes was removed by aspiration and the remaining cells were filtered through a $100-\mu \mathrm{m}$ and then a $40-\mu \mathrm{m}$ nylon strainer (BD Bioscience, Franklin Lakes, NJ, USA). Filtered cells were centrifuged for $5 \mathrm{~min}$ at $400 \mathrm{~g}$, and resuspended in $3 \mathrm{~mL}$ of growth medium [GM, containing low-glucose Dulbecco's modified Eagle's medium (LG-DMEM, Gibco, Grand Island, NY, USA) and 10\% fetal bovine serum (HyClone, Logan, UT, USA) plus 1\% antibiotic/ antimycotic]. The viability of cells was assessed using the trypan blue exclusion method and the single-cell suspension was plated onto $35-\mathrm{mm}$ culture dishes (Falcon, B\&D Bioscience, San Jose, CA, USA) at a density of 50,000 cells $/ \mathrm{cm}^{2}$. The medium was replaced $48 \mathrm{~h}$ after cell plating to remove the non-adherent red blood cells and changed twice a week thereafter.

\section{Cell colony forming and growth kinetics}

The total number of fibroblastic colony-forming units 
(CFU-F, a cluster of at least 20 adherent, fibroblast-like cells) was counted on the 7th day in primary culture. Upon reaching approximately $80-90 \%$ confluence, the cells were detached with $0.05 \%$ trypsin $/ 0.5 \mathrm{mmol} / \mathrm{L}$ EDTA (Sigma), seeded into 6-well culture plates (Falcon) at a density of 5,000 cells $/ \mathrm{cm}^{2}$, and subcultured as first-passaged cells (P1). OASCs were passaged similarly for a total of 9 passages, and the proliferative potential was determined by calculating the cumulative population doublings using the following formula: $\left[\log _{10}(\mathrm{NH})-\log _{10}(\mathrm{NI})\right] / \log _{10}(2)$, where $\mathrm{NI}$ is the inoculum cell number and $\mathrm{NH}$ is the cell harvest number. Once the cells were unable to reach confluence or a doubling time of over $100 \mathrm{~h}$ was obtained in two consecutive passages before achieving the 9th passage, the culture was considered to have failed at that passage. ${ }^{[9]}$

\section{Flow cytometry of OASCs}

The phenotypic characterization of OASCs was performed using a FACScan cytometer (Coulter Epics Altra, Becton Dickson, San Jose, CA, USA). Briefly, cells of passage 2 (P2) were trypsinized and resuspended in the flow cytometry buffer (PBS containing $0.1 \%$ FBS and $0.02 \%$ natrium azide). After blocked with human immunoglobulin, cell aliquots $\left(1 \times 10^{5}\right)$ were incubated with the following fluorescein isothiocyanate (FITC)-conjugated or phycoerythrin (PE)-conjugated monoclonal antibodies: CD14-FITC, CD19-FITC, CD34-FITC, CD45-PE, CD73-PE, CD90-FITC and CD105-FITC. Labeled cells were analyzed by flow cytometry and non-specific IgG stained cells were used as isotype controls (all antibodies from Santa Cruz Biotechnology, Dallas, TX, USA).

\section{Adipogenic differentiation of OASCs}

When OASCs of passage 3 from both young and middle-aged groups reached near-confluence, adipogenic differentiation was induced by replacing GM with the adipogenic medium (AM, consisting of GM plus $0.5 \mathrm{mmol} / \mathrm{L}$ isobutyl-methylxanthine, $10 \mathrm{mmol} / \mathrm{L}$ insulin, and $200 \mathrm{mmol} / \mathrm{L}$ indomethacin, all from Sigma). AM was changed twice a week and the intracellular lipid accumulation was assessed by oil red $O$ staining (Sigma) after 14-day differentiation. For quantification measurement, the number of oil red O-positive-staining cells was displayed as the percentage of the total cells counted within the image. Cells cultured in GM for 2 weeks served as controls.

The mRNA level of adipogenesis-related gene, peroxisome proliferator-activated receptor $\gamma(\operatorname{PPAR} \gamma)$ was quantified by real-time PCR assay. Briefly, after a 2-week adipogenic-induction, total RNA was extracted from OASCs using the RNeasy Mini Kit (Qiagen, Valencia, CA, USA). RNA samples were reverse- transcribed to cDNA using Oligo dT primers and the final cDNA was subjected to real time PCR (7300 Real-Time PCR System, Applied Biosystems, Foster City, CA, USA). Fold changes in gene expression level were calculated by the $2^{-\Delta \Delta \mathrm{Ct}}$ method and the results were normalized to the expression of an internal control, $\beta$-actin. The PCR primer sequences were listed as followed, with primer specificity confirmed on the NCBI Primer-BLAST website:

Human PPAR $\gamma$ F: 5'TCCTCGGAAATGGGACCCTC3', R: 5'ATCCACGGAGCTGATCCCAA3'.

Human $\beta$-actin F: 5'CTGGAACGGTGAAGGTGACA3', R: 5'AAGGGACTTCCTGTAACAATGCA3'.

\section{Statistical analysis}

All data collected were presented as mean \pm standard deviation. One-way analysis of variance and the Student-Newman-Keuls test were used to determine possible significant differences $(P<0.05)$ between groups.

\section{RESULTS}

\section{Morphological observation}

Grossly observed, the central fat taken from the lower lids in group $A$ had fewer blood vessels and more fibrous tissue than that in group $B$. The average mass of OF samples was $(0.412 \pm 0.189) \mathrm{g}$ and $(0.451$ $\pm 0.211) \mathrm{g}$ for the young and middle-aged groups, respectively. No significant difference was observed regarding the tissue mass between groups $(P>0.05)$. Histologically, it was found that the septal membranes separating the adipose lobules were denser in the younger group, and that the adipocytes within fatty lobules were of similar sizes and arranged tightly. In the middle-aged group, the fat cells were arranged loosely and were of not uniform size [Figure 1]. Compared to group $A$, the mean diameter, perimeter and area of OF adipocytes in group $B$ showed significantly decreasing trends $(P>0.05$, data not shown).

\section{Cell yield and growth kinetics}

The yield of viable cells isolated from OF was $(0.75$ $\pm 0.17) \times 10^{6}$ cells $/ g$ in group $A$ and $(0.68 \pm 0.13) \times$ $10^{6}$ cells $/ g$ in group $B$, with no significant difference between the two groups $(P>0.05)$. In Group $A$, the cell colonies were detected as early as $48 \mathrm{~h}$ after primary seeding and these cells proliferated rapidly. In contrast, colonies usually formed after $72 \mathrm{~h}$ or more in group $\mathrm{B}$. When the CFU-F numbers were determined on the 7th day in culture, more and bigger colonies were observed in group A than in group B (19.45 \pm 2.52 vs. $8.42 \pm 1.37, P<0.001$ ) [Figure 2]. Passaged cells from both groups exhibited similar homogeneous fibroblast-like morphology, but the doubling time in 

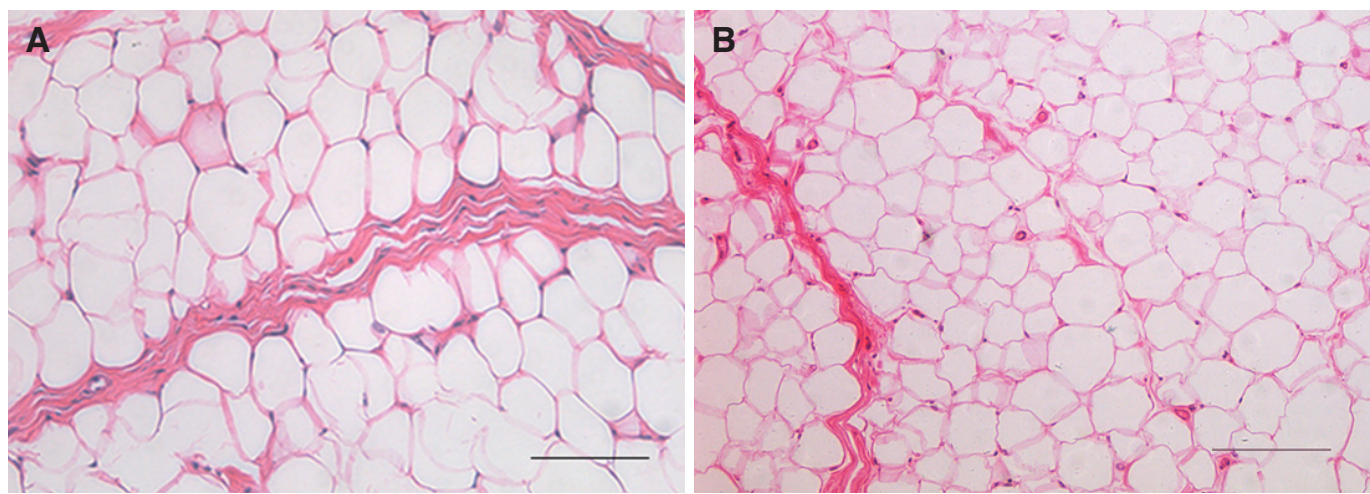

Figure 1: Representative HE staining images of the orbital fat samples from young donors (A) and middle-aged donors (B). The adipocytes were smaller and fibrous structure appeared looser in $(B)$ than those in $(A)($ scale bar: $100 \mu \mathrm{m})$
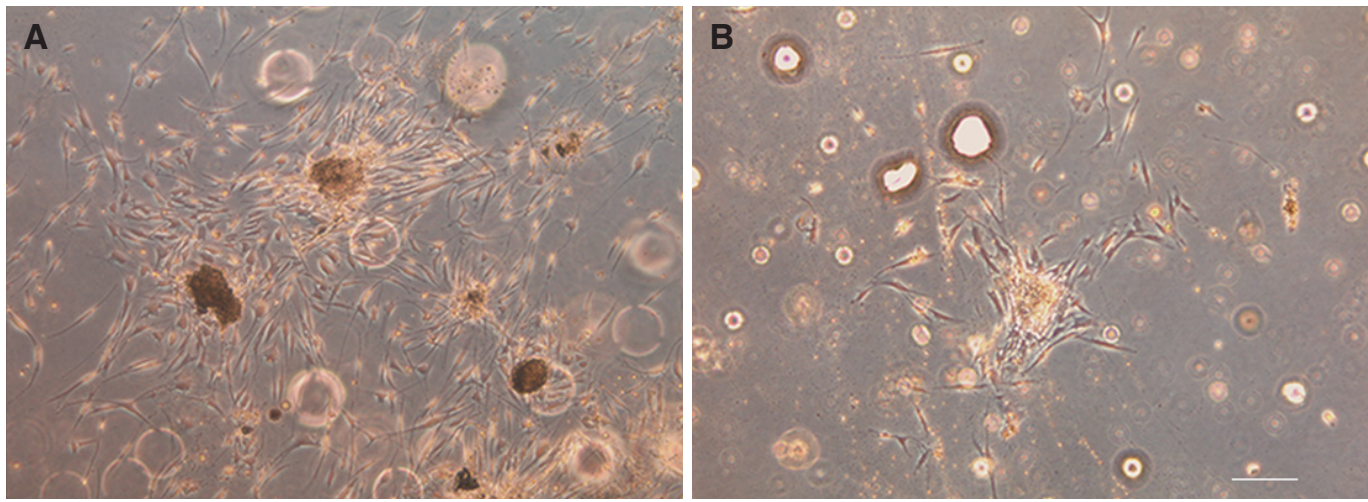

Figure 2: The colony formation of orbital adipose-derived stem cells was determined at day 7 in the primary culture. Compared to middleaged donors (B), both the colony number and the cell number within each colony were greater in young donors (A) (scale bar: $100 \mu \mathrm{m})$

group B was significantly longer than that of group A $(P<0.05$, data not shown). Four of 6 cell lines in group $B$ ceased to proliferate at the 6th passage, and only two reached the 7 th passage. In contrast, in group A, 5 cell lines from the 6 donors reached the 9th passage and 1 failed at the 7 th passage. Thus the cell population doublings (CPDs) were compared in pairs from $\mathrm{P} 1$ to $\mathrm{P} 5$. On average OASCs in group A attained 13.41 CPDs. In contrast, only 8.7 doublings were achieved in group $\mathrm{B}(P<0.001)$.

\section{Flow cytometry analysis}

The cell surface marker expression of OASCs from older and younger donors was compared using flow cytometry analysis. Our results showed that cells in both groups were negative for CD14, CD19, CD34 and CD45, and positive for CD73, CD90 and CD105 (typical markers for adult mesenchymal stem cells, MSCs). No significant difference existed between the two groups $(P>0.05)$.

\section{Adipogenic differentiation potential}

Adipogenic differentiation of OASCs was confirmed by oil red $\mathrm{O}$ staining after a 14-day induction period. Compared to the non-induced cells, which exhibited long spindle-like morphology and failed to accumulate any lipid, differentiating OASCs assumed an expanded morphology consistent with adipocytes and contained red staining lipid droplets within the cytoplasm [Figure 3]. Quantitatively, the percentage of oil red O-positive staining cells in group A was obviously higher than that in group $\mathrm{B}(P<0.001)$ [Figure 4A]. Consistent with the staining findings, the real-time PCR results revealed a significantly lower expression level of PPAR $\gamma$ mRNA in the middle-aged group than in the younger group $(P<0.001)$ [Figure 4B].

\section{DISCUSSION}

The multi-lineage differentiation capacity of OASCs has shown great therapeutic potential in the fields of ophthalmology and regenerative medicine. ${ }^{[4-7]}$ Although the literature indicates that aging has less effect on the self-renewal and differentiation potential of ASCs isolated from SF tissue ${ }^{[10-12]}$ whether this fact holds true in OASCs derived from elderly humans remains unknown. In this study, the authors make the first report on the age-related effects on the biological properties of human OASCs, demonstrating that the adipogenic differentiation and proliferative capabilities of OASCs decrease with advancing age. 

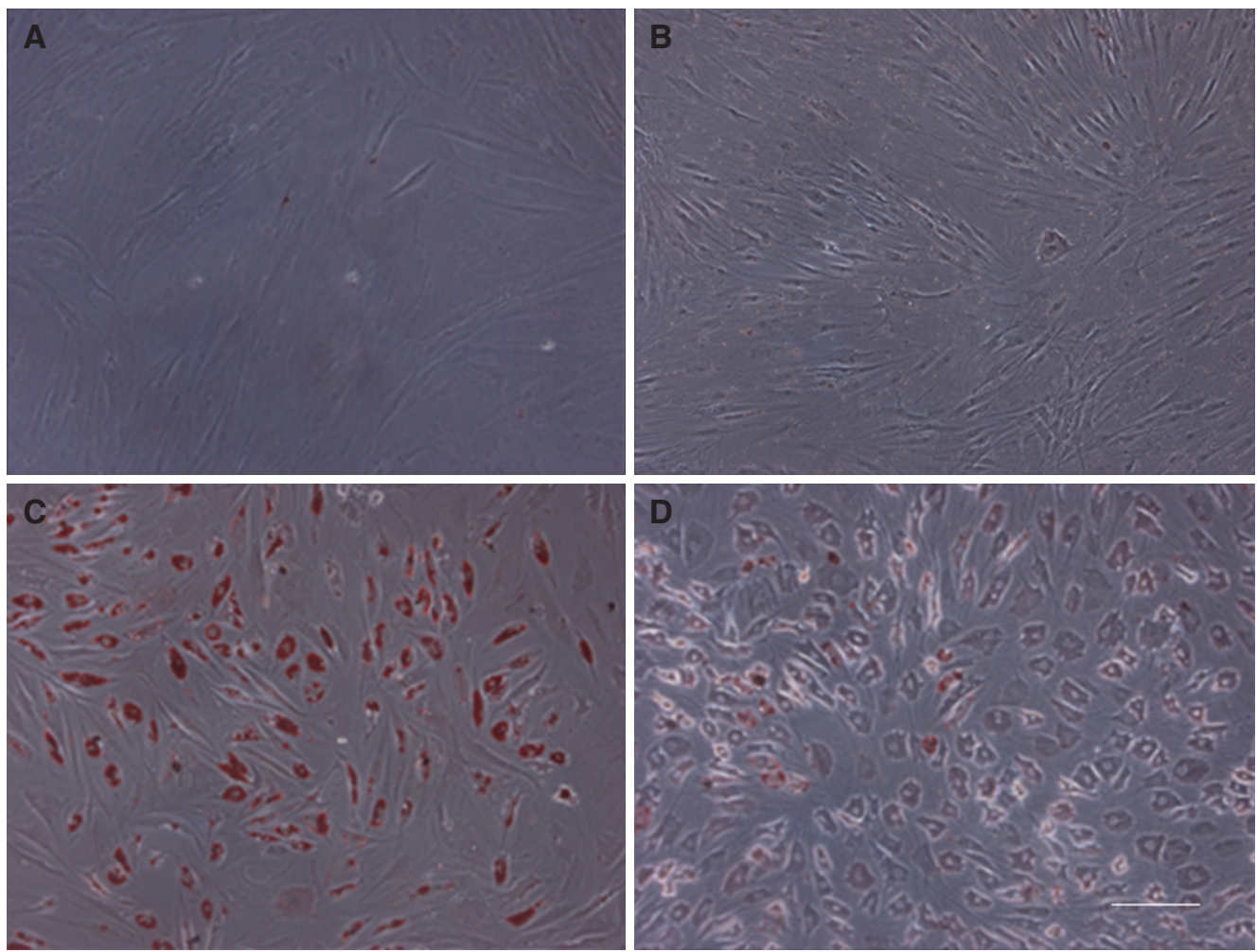

Figure 3: Adipogenic differentiation was assessed by oil red $\mathrm{O}$ staining after culturing orbital adipose-derived stem cells in adipogenic media for 14 days. (A, C): young group; $(B, D)$ : middle-aged group; $(A, B)$ : non-induced; $(C, D)$ : induced. More positive staining cells were observed in $(A, C)$ than those in $(B, D)$, and almost no staining was seen in $(A, B)$ (scale bar: $100 \mu m)$

A

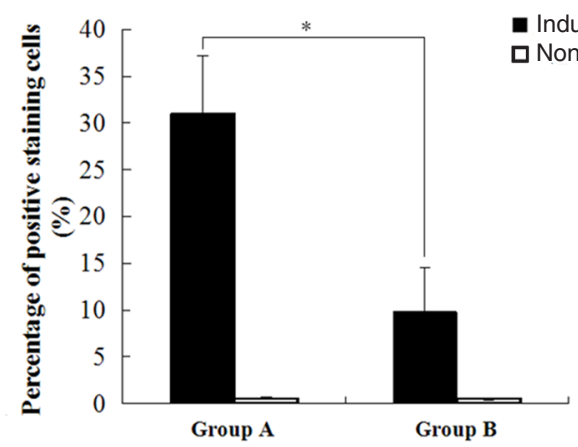

B

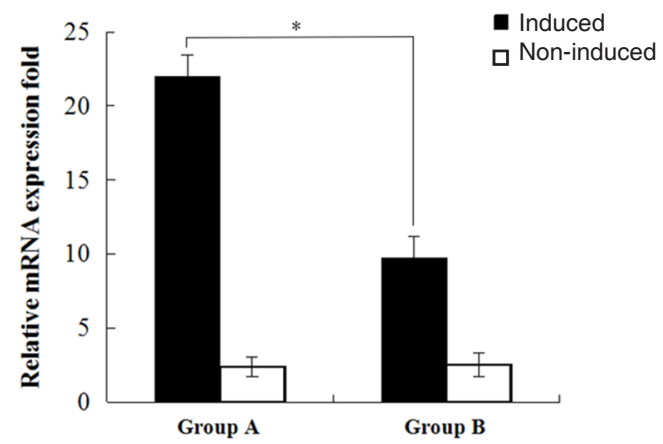

Figure 4: Quantitative analysis confirmed the adipogenic differentiation of orbital adipose-derived stem cells in contrast with the noninduced cells. Compared to the young age group, both the percentage of oil red $O$ staining-positive cells (A) and the expression level of peroxisome proliferator-activated receptor $\gamma$ mRNA $(B)$ were significantly reduced in the middle-aged group $\left({ }^{\star} P<0.001\right)$

OF is derived from both mesodermal and ectodermal cells. ${ }^{[13]}$ The central fat pads of both upper and lower lids are mesodermally derived, while the medial fat in the upper and lower eyelids develops from the neural crest cells. ${ }^{[4,5]}$ Therefore, to achieve consistency between groups, all samples used in this study were taken from the central fat compartments of the lower eyelids.

At the histological level, the fibrous septum surrounding the fat lobules was thin and sparse in the middle-aged group, and the loosely arranged adipocytes appeared smaller than those in the younger age group. Changes of septal structure correlated with age were predicted, and were attributable to OF herniation, resulting in a baggy-eye appearance. Irregular and decreased fat cell size at middle age may indicate the reduced capacity to accumulate lipid and reserve energy. ${ }^{[14]}$

Next, OASCs were isolated and expanded in vitro. The cell phenotype was characterized by flow cytometry, and the effects of age on the number of OASCs', their proliferation, and differentiation were investigated. OASCs from both groups expressed a similar CD marker profile, which was consistent with that reported for ASCs from SF depots. Although the viable cells 
freshly isolated from OF were comparable between two groups, the progenitor cell numbers declined in the older group. Because some cell lines ceased to grow at passage 6 , the cell growth kinetics were compared from passages 1 to 5 . It was found that the proliferation rate of young OASCs was significantly greater than that of the middle-aged cells. Young OASCs could generally achieve more than 12 population doublings while 10 CPDs were the maximum that cells from middle-aged donors could achieve. Moreover, an agerelated decrease in the adipogenic-differentiation potential of OASCs was observed. Not only the percentage of oil red $O$ staining-positive cells but also the mRNA expression of PPAR $\gamma$, a key transcription factor regulating adipogenic differentiation and maintaining fat cell phenotype, declined with age.

In general, our data showed that aging had a great influence on the proliferation and differentiation potentials of OASCs. The OASCs' number, adipogenic differentiation and proliferative potentials began to decrease at middle or early old age, which may render their use in autologous cell therapy unsuitable in elderly patients. Whether other linage differentiation capabilities of OASCs are correlated with age remains to be investigated in further studies. On the other side, our previous studies proved that ASCs isolated from SF depots possess low immunogenicity and can maintain their biological functions after cryopreservation. ${ }^{[15,16]}$ If these properties prove true in OASCs, allogenic or cryopresereved autologous OASCs may serve as alternatives for future clinical applications in elderly patients.

\section{Financial support and sponsorship}

This work was financially supported by the grants of National Natural Science Foundation of China (No. 31271027 and No. 81171475).

\section{Conflicts of interest}

There are no conflicts of interest.

\section{Patient consent}

All patients had previously given informed consent.

\section{Ethics approval}

The protocol for this study was approved by the Research Ethics Committee of Tongji University School of Medicine (No. 2015-0083) and conformed to the principles outlined in the Declaration of Helsinki.

\section{REFERENCES}

1. De Francesco F, Ricci G, D’Andrea F, Nicoletti GF, Ferraro GA. Human adipose stem cells: from bench to bedside. Tissue Eng Part B Rev 2015;21:572-84.

2. Oedayrajsingh-Varma MJ, van Ham SM, Knippenberg M, Helder MN, Klein-Nulend J, Schouten TE, Ritt MJ, van Milligen FJ. Adipose tissue-derived mesenchymal stem cell yield and growth characteristics are affected by the tissue-harvesting procedure. Cytotherapy 2006;8:166-77.

3. Schipper BM, Marra KG, Zhang W, Donnenberg AD, Rubin JP Regional anatomic and age effects on cell function of human adipose-derived stem cells. Ann Plast Surg 2008;60:538-44.

4. Korn BS, Kikkawa DO, Hicok KC. Identification and characterization of adult stem cells from human orbital adipose tissue. Ophthal Plast Reconstr Surg 2009;25:27-32.

5. Chen SY, Mahabole M, Horesh E, Wester S, Goldberg JL, Tseng $\mathrm{SC}$. Isolation and characterization of mesenchymal progenitor cells from human orbital adipose tissue. Invest Ophthalmol Vis Sci 2014;55:4842-52.

6. Ho JH, Ma WH, Tseng TC, Chen YF, Chen MH, Lee OK. Isolation and characterization of multi-potent stem cells from human orbital fat tissues. Tissue Eng Part A 2011;17:255-66.

7. Lin KJ, Loi MX, Lien GS, Cheng CF, Pao HY, Chang YC, Ji AT, Ho JH. Topical administration of orbital fat-derived stem cells promotes corneal tissue regeneration. Stem Cell Res Ther 2013;4:72.

8. Bujalska IJ, Durrani OM, Abbott J, Onyimba CU, Khosla P, Moosavi AH, Reuser TT, Stewart PM, Tomlinson JW, Walker EA, Rauz S. Characterisation of $11 \beta$-hydroxysteroid dehydrogenase 1 in human orbital adipose tissue: a comparison with subcutaneous and omental fat. J Endocrinology 2007;192:279-88.

9. Lin TM, Tsai JL, Lin SD, Lai CS, Chang CC. Accelerated growth and prolonged lifespan of adipose tissue-derived human mesenchymal stem cells in a medium using reduced calcium and antioxidants. Stem Cells Dev 2005;14:92-102.

10. Mendes SC, Tibbe JM, Veenhof M, Bakker K, Both S, Platenburg PP, Oner FC, de Bruijn JD, van Blitterswijk CA. Bone tissueengineered implants using human bone marrow stromal cells: effect of culture conditions and donor age. Tissue Eng 2002;8:911-20.

11. Chen HT, Lee MJ, Chen CH, Chuang SC, Chang LF, Ho ML, Hung SH, Fu YC, Wang YH, Wang HI, Wang GJ, Kang L, Chang JK. Proliferation and differentiation potential of human adiposederived mesenchymal stem cells isolated from elderly patients with osteoporotic fractures. J Cell Mol Med 2012;16:582-93.

12. Zhu M, Kohan E, Bradley J, Hedrick M, Benhaim P, Zuk P. The effect of age on osteogenic, adipogenic and proliferative potential of female adipose-derived stem cells. J Tissue Eng Regen Med 2009;3:290-301.

13. Billon N, Monteiro MC, Dani C. Developmental origin of adipocytes: new insights into a pending question. Biol Cell 2008;100:563-75.

14. Kirkland JL, Dobson DE. Preadipocyte function and aging: links between age-related changes in cell dynamics and altered fat tissue function. J Am Geriatr Soc 1997;45:959-67.

15. Liu G, Zhou H, Li Y, Li G, Cui L, Liu W, Cao Y. Evaluation of the viability and osteogenic differentiation of cryopreserved human adipose-derived stem cells. Cryobiology 2008;57:18-24.

16. Liu G, Zhang Y, Liu B, Sun J, Li W, Cui L. Bone regeneration in a canine cranial model using allogeneic adipose derived stem cells and coral scaffold. Biomaterials 2013;34:2655-64. 